\title{
A three-year survey of the antimicrobial resistance of microorganisms at a Chinese hospital
}

\author{
JING TANG $^{1}$, LILI WANG ${ }^{2}$, YUFEI XI $^{1}$ and GAOLIN LIU ${ }^{1}$ \\ Departments of ${ }^{1}$ Clinical Pharmacy and ${ }^{2}$ Blood Transfusion, Shanghai First People's Hospital, School of Medicine, \\ Shanghai Jiaotong University, Shanghai 200080, P.R. China
}

Received February 10, 2015; Accepted October 13, 2015

DOI: $10.3892 /$ etm.2016.2983

\begin{abstract}
The present retrospective study aimed to investigate the antibiotic susceptibility of bacteria at Shanghai First People's Hospital (Shanghai, China) between 2009 and 2011. An increasing trend of antibiotic resistance was observed in this hospital between 2009 and 2011. Escherichia coli, Acinetobacter baumannii and Staphylococcus aureus were the most prevalent resistant strains. Antimicrobial susceptibility was detected using standard Kirby-Bauer disk diffusion and analyzed using World Health Organization software. E. coli was demonstrated to be the most prevalent bacterium in the present survey between 2009 and 2011 (16.2, 20.0 and $19.6 \%$, respectively); followed by A. baumannii $(13.5,13.3$ and $10.6 \%$, respectively) and $S$. aureus. Notably, $>70 \%$ of $E$. coli and $70 \%$ of $S$. aureus were resistant to common antibiotics; whereas $60 \%$ of A. baumannii and $20 \%$ of Pseudomonas aeruginosa were resistant to the majority of the antibiotics investigated. In 2011, Enterococcus faecalis exhibited a resistance rate of $55.6 \%$ against levofloxacin and E. faecium exhibited a $53.2 \%$ resistance rate. The present survey demonstrated an increasing trend in bacterial resistance against antibiotics; therefore, more stringent guidelines for antibiotics should be advocated.
\end{abstract}

\section{Introduction}

Surveillance of antibiotic resistance is the most effective method for obtaining accurate data regarding bacterial resistance transitions and epidemiology. This data informs the formulation of guidelines for the use of antibiotics, and helps control the spread of resistant bacteria (1). In recent years, microbial resistance to antibiotics has been increasing,

Correspondence to: Dr Gaolin Liu, Department of Clinical Pharmacy, Shanghai First People's Hospital, School of Medicine, Shanghai Jiaotong University, 100 Haining Road, Shanghai 200080, P.R. China

E-mail: angel_tj99@163.com

Key words: Escherichia coli, bacterial infection, antimicrobial resistance, Acinetobacter, Staphylococcus aureus particularly in China (2-5). If this trend continues there will be fewer antibiotics to choose from when treating patients $(6,7)$. Formulating policy for the use of antibiotics and the improved management of patients depends on up-to-date knowledge of the prevalent strains of bacteria in particular locales or regions of a country, and their likely patterns of antibiotic resistance. To this end, in the present retrospective study, enabled by the routine practice of monitoring clinical bacterial isolates and recording corresponding antibiograms, the clinical bacterial profiles of patients were collected and analyzed between 2009 and 2011 at Shanghai First People's Hospital (Shanghai, China). Patterns of antibiotic resistance were determined in order to ascertain an accurate representation of local bacterial resistance and to help improve the efficacy of empirical antibiotic therapy.

\section{Materials and methods}

Clinical survey. The present study retrospectively investigated the antibiotic susceptibility of bacteria between 2009 and 2011 at Shanghai First People's Hospital affiliated with Shanghai Jiaotong University. The study included a total of 5,209 patients (63\% male), from whom were collected sputum, blood and wound culture samples. The present study was approved by the ethics committee of Shanghai First People's Hospital. Written informed consent was obtained from all participants.

Bacterial strains and tests for antimicrobial susceptibility. The quality control bacterial strains included Staphylococcus aureus (ATCC25923), Escherichia coli (ATCC25922), Pseudomonas aeruginosa (ATCC27853), Enterococcus faecalis (ATCC29212), and Klebsiella pneumoniae (ATCC700603; Shanghai Center for Clinical Laboratory, Shanghai, China). Mueller-Hinton agar, which was used for susceptibility testing, and antibiotic susceptibility disks were purchased from Oxoid (Thermo Fisher Scientific Inc., Waltham, MA, USA). Antimicrobial susceptibility testing data were generated as part of a surveillance program conducted by the Shanghai First People's Hospital between 2009 and 2011. Drug susceptibility tests were performed using the Kirby-Bauer antibiotic testing disk diffusion method (8), and the data were interpreted in accordance with the 2010 guidelines outlined by the Clinical and Laboratory Standards Institute (8). 


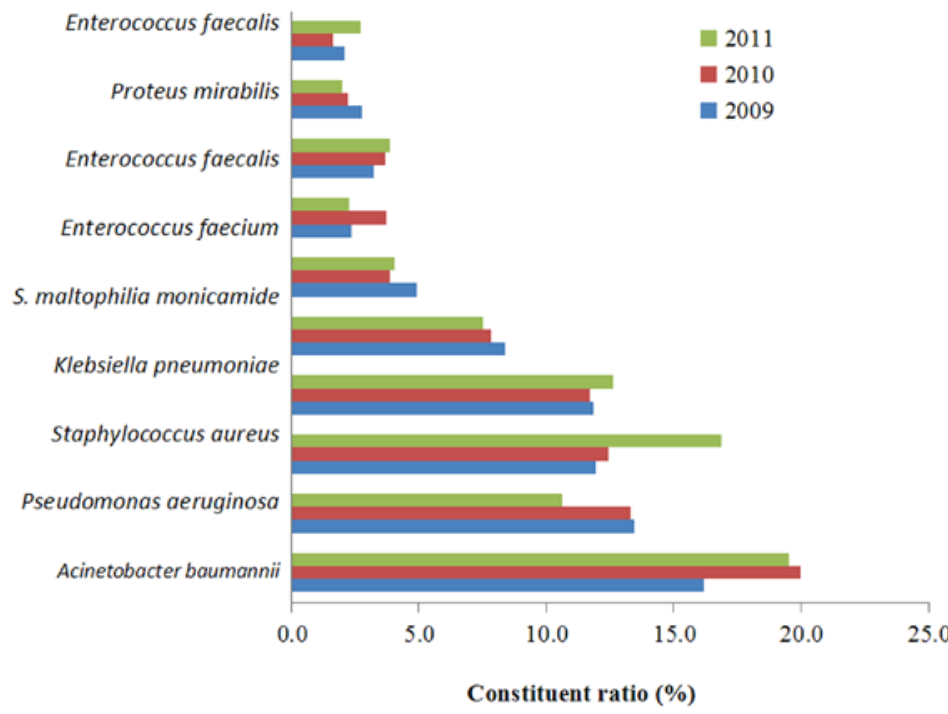

Figure 1. Prevalence of bacterial pathogens at Shanghai First People's Hospital (Shanghai, China) between 2009 and 2011.
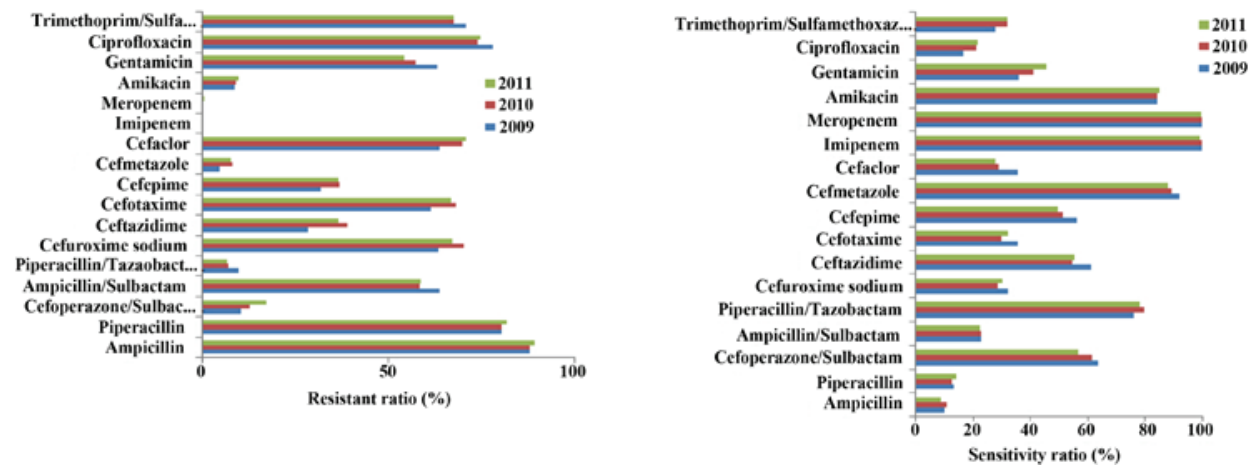

Figure 2. Resistance and sensitivity rates of Escherichia coli to penicillins, including ampicillin, piperacillin and ampicillin/sulbactam, cephalosporins and other antibiotics between 2009 and 2011.

Statistical analyses. WHONET 5.5 software, which was provided by the Antimicrobial Resistance Monitoring Center of the World Health Organization (Geneva, Switzerland), was utilized for statistical analyses.

\section{Results}

Distribution of the pathogenic bacteria. Between 2009 and 2011, E. coli was the most prevalent bacterial pathogen detected in the present hospital, with the following detection rates: 2009, 305/1883 (16.2\%); 2010, 346/1730 (20\%); 201, 408/2082 (19.6\%) (Fig. 1). The next most prevalent was Acinetobacter baumannii: 2009, 225/1875 (12.0\%); 2010, 216/1728 (12.5); and 2011, 253/1497 (16.9\%), and S. aureus was the third most prevalent pathogenic bacterium by 2011 .

Sensitivity and resistance rates of the bacterial strains $E$. coli. Susceptibility testing of $E$. coli indicated $>60 \%$ resistance against the following antibiotics: Penicillins, including ampicillin, piperacillin and ampicillin/sulbactam; certain cephalosporins, including cefuroxime, cefotaxime and cefaclor; and other antibiotics, including ciprofloxacin, trimethoprim, sulphamethoxazole and gentamicin. Imipenem and meropenem were demonstrated to be the most effective antibiotics against $E$. coli. By 2011, the resistance rate of $E$. coli against meropenem had increased to $0.2 \%$ and since then the trend for resistance against imipenem and meropenem has increased, with the susceptibility break point increasing from $14-15$ to 20-22 (Fig. 2).

S. aureus. The resistance of $S$. aureus to the most commonly used antibiotics, including: Penicillin, cefazolin, gentamicin, clindamycin, erythromycin and levofloxacin, was demonstrated to be $>70 \%$. S. aureus exhibited minimal resistance to linezolid, vancomycin and teicoplanin in the three consecutive years (Fig. 3).

A. baumannii. For the majority of the antibiotics investigated, the resistance rates of $A$. baumannii were $>60 \%$. Cefoperazone/sulbactam was demonstrated to be the most effective antibiotic treatment against this bacterium; however, its resistance rate increased from 26.1 to $44.6 \%$ between 2009 and 2011 (Fig. 4).

P. aeruginosa. For the majority of the antibiotics investigated against $P$. aeruginosa, the resistance rates were $\sim 20 \%$, including cefoperazone/sulbactam and piperacillin, whereas the resistance rates against amikacin were $\sim 10 \%$ (Fig. 5). 


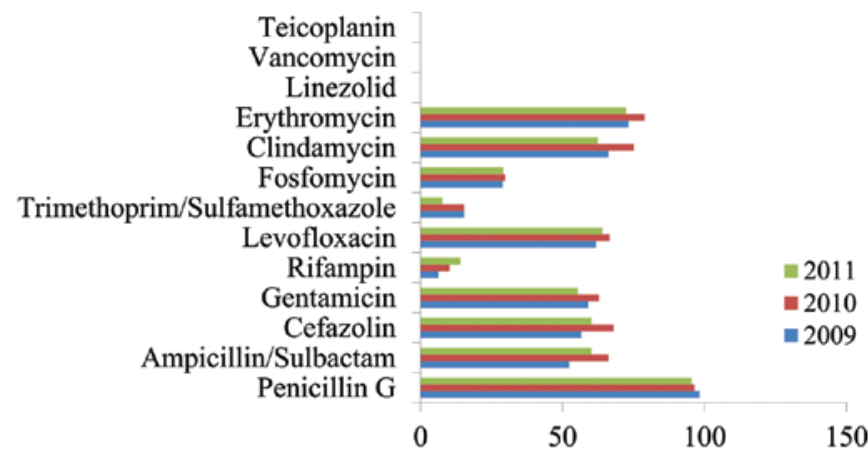

Resistance ratio (\%)

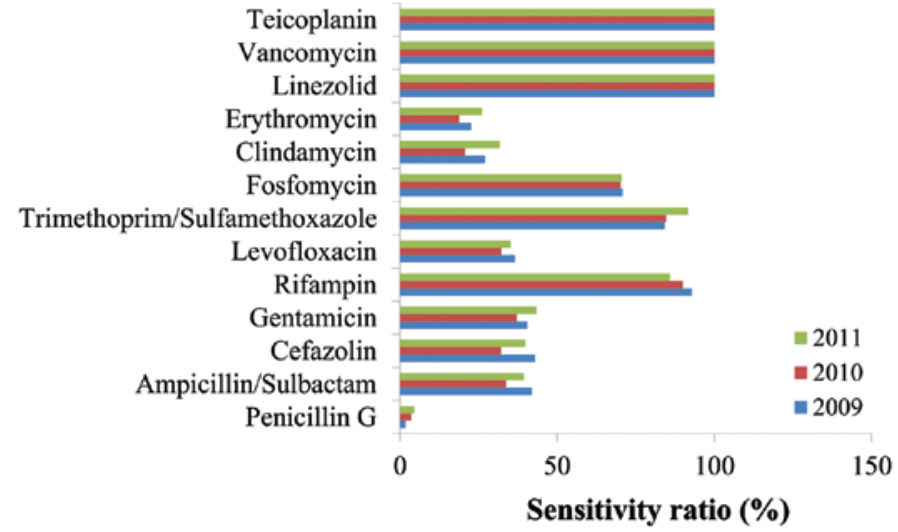

Figure 3. Resistance and sensitivity rates of Staphylococcus aureus to common antibiotics: Penicillin, cefazolin, gentamicin, clindamycin, erythromycin, levofloxacin, linezolid, vancomycin and teicoplanin between 2009 and 2011

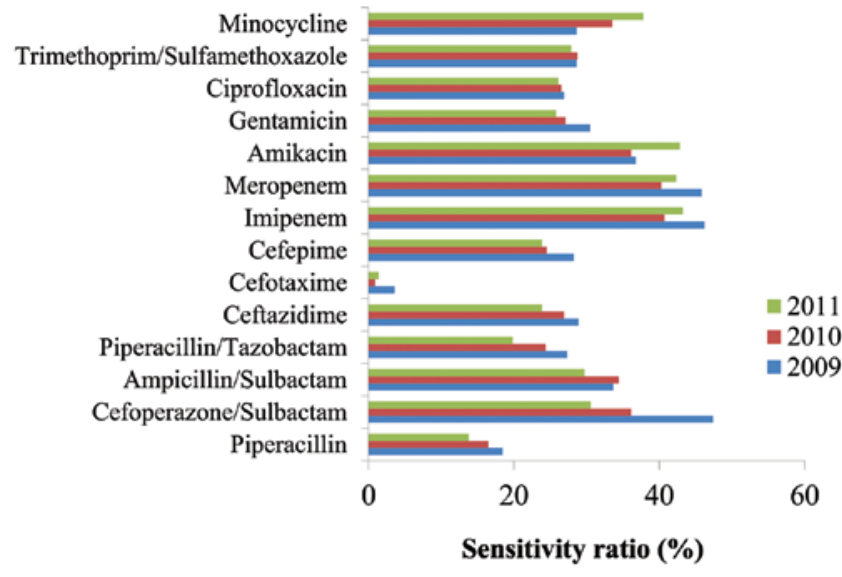

Figure 4. Resistance and sensitivity rates of Acinetobacter baumannii to various antibiotics between 2009 and 2011.
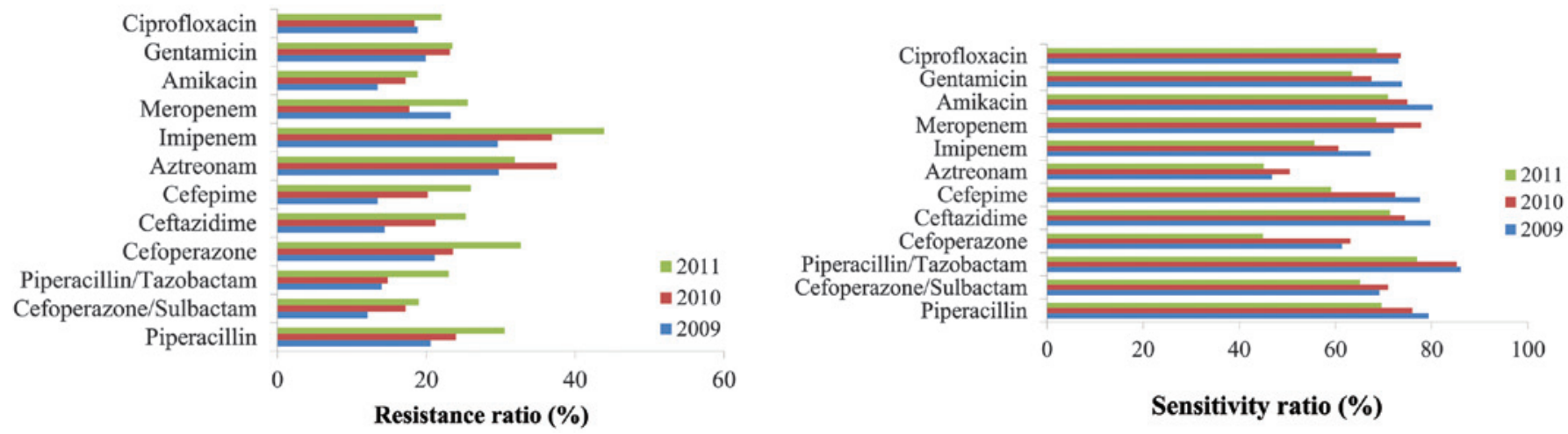

Sensitivity ratio (\%)

Figure 5. Resistance and sensitivity rates of Pseudomonas aeruginosa to various antibiotics between 2009 and 2011.

K. pneumoniae. With sensitivity rates of $100 \%$ to imipenem and meropenem in 2009 and 2010, K. pneumoniae was the most highly sensitive bacterium; however, $K$. pneumoniae had developed a small degree of resistance to these antibiotics by 2011 (Fig. 6).

Stenotrophomonas maltophilia monicamide. S. maltophilia monicamide was demonstrated to be sensitive to cefoperazone/sulbactam (Fig. 7), with no change demonstrated over the three years.
E. faecalis. In 2011, E. faecalis exhibited a resistance rate of $55.6 \%$ against levofloxacin, which was a notable increase compared with previous years. E. faecalis was demonstrated to be $100 \%$ sensitive to linezolid, vancomycin and teicoplanin (Fig. 8).

Proteus. mirabilis. P. mirabilis was initially $100 \%$ sensitive to cefoperazone/sulbactam, imipenem, meropenem and gentamicin; however, this sensitivity decreased by $0.0,21.4,4.8$ and $31.0 \%$, respectively, over three years (Fig. 9). 

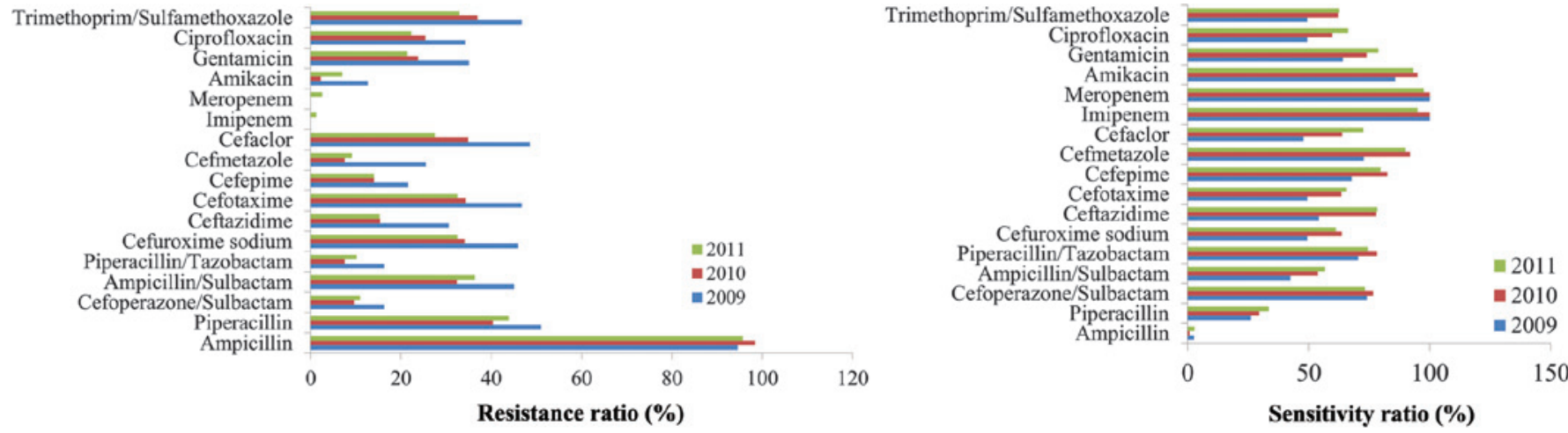

Figure 6. Resistance and sensitivity rates of Klebsiella pneumoniae to various antibiotics between 2009 and 2011.
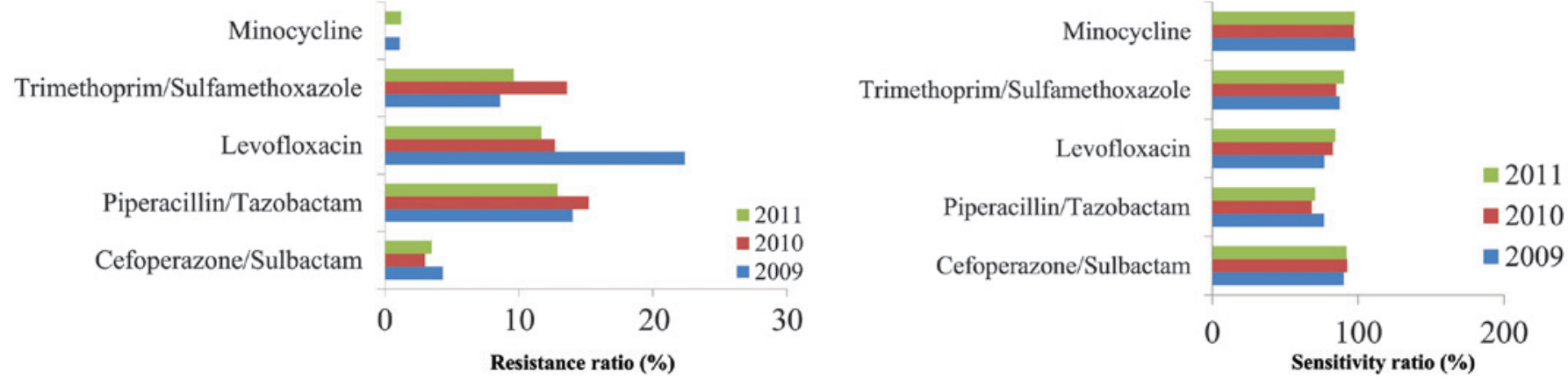

Figure 7. Resistance and sensitivity rates of Stenotrophomonas maltophilia monicamide to various antibiotics between 2009 and 2011.
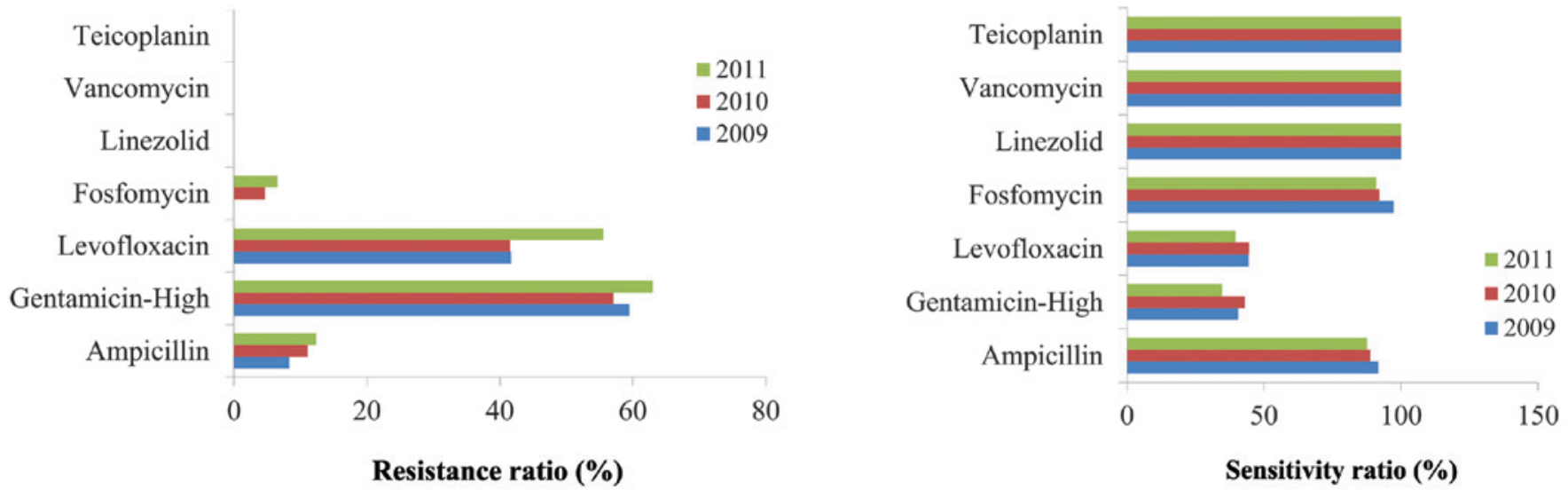

Figure 8. Resistance and sensitivity rates of Enterococcus faecalis to various antibiotics between 2009 and 2011.

Methicillin-resistant S. aureus. Between 2009 and 2011, the detection rates for methicillin-resistant $S$. aureus were 63.2, 65.8 and $60.1 \%$, respectively (Fig. 10); whereas the detection rates for methicillin-resistant coagulase-negative staphylococci were $68.0,71.6$ and $68.5 \%$, respectively between 2009 and 2011.

\section{Discussion}

The results of the present study demonstrated that the most common pathogen detected between 2009 and 2011 at Shanghai First People's Hospital was E. coli, followed by A. baumannii, $P$. aeruginosa, $S$. aureus, $K$. pneumoniae, S. maltophilia, E. faecium, E. faecalis, P. mirabilis and E. cloacae. The percentage of Gram-negative bacteria detected was significantly higher, as compared with that of Gram-positive bacteria. Furthermore, non-fermenting Gram-negative bacteria were more prevalent than Enterobacteriaceae, and A. baumannii was more prevalent than $P$. aeruginosa, which was demonstrated to be the most prevalent pathogen at the hospital since 2011 .

At present, antibiotic consumption in China is high, with various quantities and classes of antibiotics being prescribed, depending on the type of hospital (9-11). Given that the worldwide spread of multidrug-resistant bacteria is threatening the availability of safe and effective antibiotic treatment for patients (12), strong infection control measures and alternative therapeutic agents are required. Methicillin-resistant $S$. aureus remains the primary Gram-positive bacterium of concern in 

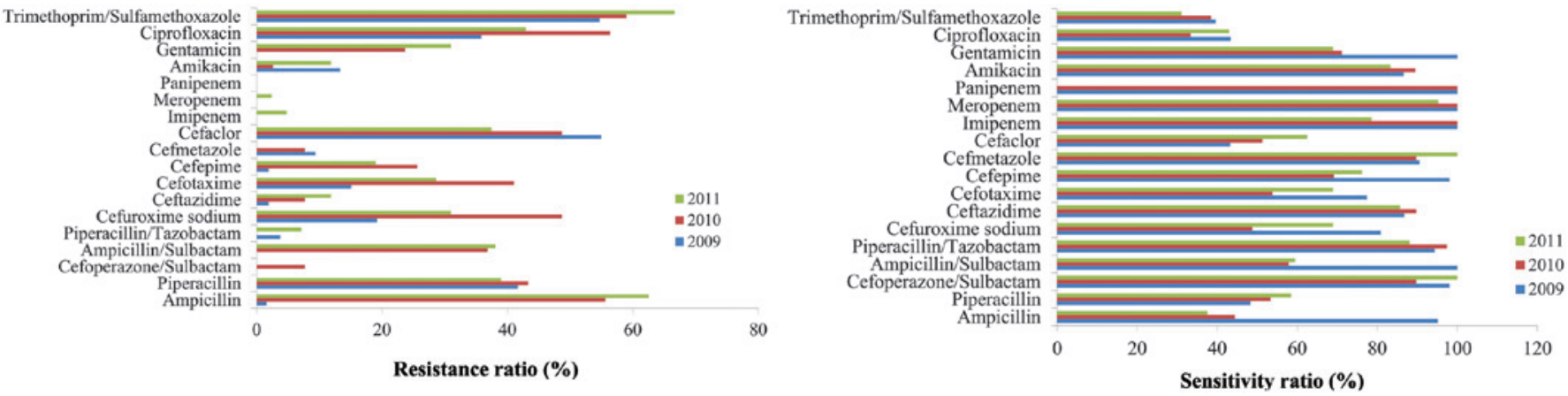

Figure 9. Resistance and sensitivity rates of Proteus mirabilis to various antibiotics between 2009 and 2011.

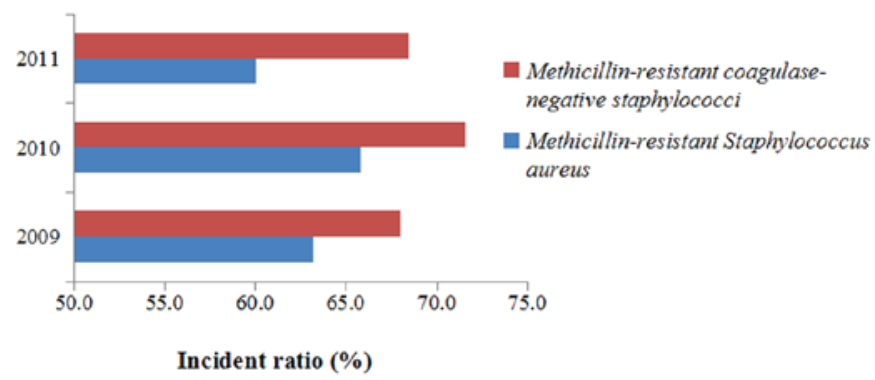

Figure 10. Detection rates of methicillin-resistant Staphylococcus aureus and methicillin-resistant coagulase-negative staphylococci between 2009 and 2011.

public hospitals $(13,14)$. Strains of this bacterium are resistant to $\beta$-lactams, macrolides, fluoroquinolones and aminoglycosides; therefore, glycopeptide antibacterial agents remain the last line of antimicrobial defense.

Antibiotic consumption and the development of bacterial resistance are closely associated, and antibiotic resistance rates are increasing in developing countries, as compared with those in developed countries (15). Extensive use of third-generation cephalosporins has caused bacteria under this selective pressure to generate extended-spectrum $\beta$-lactamases and AmpC enzymes, which are capable of overcoming the anti-bacterial activities of cephalosporins (16). In the present study, the susceptibility tests demonstrated that the majority of bacteria were highly sensitive to linezolid, vancomycin and teicoplanin; therefore, these remain effective antimicrobial agents against otherwise resistant bacteria. Furthermore, the present study demonstrated that Gram-negative bacilli may be more sensitive to imipenem. All bacteria were $100 \%$ sensitive to imipenem and meropenem in 2009 and 2010; however, a small increase in resistance to imipenem was detected in 2011.

In order to mitigate the development of antibiotic resistance and reduce nosocomial infections, clinicians should pay particular attention to clinical indications. Furthermore, clinicians should understand the dynamics of bacterial resistance, the results of bacterial susceptibility tests, and develop appropriate prevention measures that seek to reduce the incidence of nosocomial infections and the spread of resistant bacteria $(17,18)$. E. coli remains the most common pathogen at Shanghai First People's Hospital, and the resistance rates of bacteria to conventional and emerging antibiotics are increasing. Previous studies investigating similar control strategies supported the effectiveness of targeted active surveillance (19-21). The present study suggested that clinicians should seek to prescribe medications based on antimicrobial susceptibility results, in order to avoid the misuse of antibiotics and to reduce the probability of resistant strains emerging under the selection pressure.

\section{Acknowledgements}

This study was supported by grants from the Natural Science Foundation of China (No. 81470852), the Science and Technology Commission of Shanghai Science and Technology Support Project (No. 13431900503), the Medical and Technology Across Project of Shanghai Jiaotong University (No. YG2012MS02) and Young Talents Project of Shanghai Health System (No. XYQ2013091).

\section{References}

1. Linder JA, Bates DW and Platt R: Antivirals and antibiotics for influenza in the United States, 1995 - 2002. Pharmacoepidemiol Drug Saf 14: 531-536, 2005.

2. Xie DS, Xiong W, Lai RP, Liu L, Gan XM, Wang XH, Wang M, Lou YX, Fu XY, Wang HF, et al: Ventilator-associated pneumonia in intensive care units in Hubei Province, China: A multicentre prospective cohort survey. J Hosp Infect 78: 284-288, 2011.

3. Chuang JH, Huang AS, Huang WT, Liu MT, Chou JH, Chang FY and Chiu WT: Nationwide surveillance of influenza during the pandemic (2009-10) and post-pandemic (2010-11) periods in Taiwan. PLoS One 7: e36120, 2012.

4. You JH,ChanES,Leung MY,Ip Mand Lee NL: A cost-effectiveness analysis of "test" versus "treat" patients hospitalized with suspected influenza in Hong Kong. PLoS One 7: e33123, 2012.

5. Lee $\mathrm{N}$ and Ison MG: Diagnosis, management and outcomes of adults hospitalized with influenza. Antivir Ther 17 (1 Pt B): $143-157,2012$.

6. Linder JA, Chan JC and Bates DW: Appropriateness of antiviral prescribing for influenza in primary care: A retrospective analysis. J Clin Pharm Ther 31: 245-252, 2006.

7. Sarkar P and Gould IM: Antimicrobial agents are societal drugs: How should this influence prescribing? Drugs 66: 893-901, 2006.

8. Wayne P: Performance standards for antimicrobial susceptibility testing. Ninth informational supplement NCCLS document M100-S9. National Committee for Clinical Laboratory Standards, 2008.

9. Hvistendahl M: Public health. China takes aim at rampant antibiotic resistance. Science 336: 795-795, 2012.

10. Li Y, Xu J, Wang F, Wang B, Liu L, Hou W, Fan H, Tong Y, Zhang $\mathrm{J}$ and $\mathrm{Lu} \mathrm{Z}$ : Overprescribing in China, driven by financial incentives, results in very high use of antibiotics, injections, and corticosteroids. Health Aff (Millwood) 31: 1075-1082, 2012.

11. Song W, Cao J and Mei YL: Correlation between cephamycin consumption and the incidence of antimicrobial resistance in Acinetobacter baumannii at a university hospital in China from 2001 to 2009. Int J Clin Pharmacol Ther 49: 765-771, 2011. 
12. Amadeo B, Dumartin C, Venier A, Fourrier-Réglat A, Coignard B and Rogues AM: Factors associated with the prevalence of antibiotic use for the treatment of hospital-acquired infections at 393 French hospitals: A regional variation analysis. Infect Control Hosp Epidemiol 32: 155-162, 2011.

13. Collins J, Raza M, Ford M, Hall L, Brydon S and Gould FK Review of a three-year meticillin-resistant Staphylococcus aureus screening programme. J Hosp Infect 78: 81-85, 2011.

14. Waterhouse M, Morton A, Mengersen K, Cook D and Playford G: Role of overcrowding in meticillin-resistant Staphylococcus aureus transmission: Bayesian network analysis for a single public hospital. J Hosp Infect 78: 92-96, 2011.

15. Gupta A, Kapil A, Lodha R, Kabra SK, Sood S, Dhawan B, Das BK and Sreenivas V: Burden of healthcare-associated infections in a paediatric intensive care unit of a developing country: A single centre experience using active surveillance. J Hosp Infect 78: 323-326, 2011.

16. Kumarasamy KK, Toleman MA, Walsh TR, Bagaria J, Butt F, Balakrishnan R, Chaudhary U, Doumith M, Giske CG, Irfan S, et al: Emergence of a new antibiotic resistance mechanism in India, Pakistan, and the UK: A molecular, biological, and epidemiological study. Lancet Infect Dis 10: 597-602, 2010.

17. Bouzbid S, Gicquel Q, Gerbier S, Chomarat M, Pradat E, Fabry J, Lepape A and Metzger MH: Automated detection of nosocomial infections: Evaluation of different strategies in an intensive care unit 2000-2006. J Hosp Infect 79: 38-43, 2011.
18. Yang $\mathrm{M}, \mathrm{Hu} \mathrm{Z}$ and $\mathrm{Hu} \mathrm{F}$ : Nosocomial meningitis caused by Acinetobacter baumannii: Risk factors and their impact on patient outcomes and treatments. Future Microbiol 7: 787-793, 2012.

19. Harbarth S, Hawkey PM, Tenover F, Stefani S, Pantosti A and Struelens MJ: Update on screening and clinical diagnosis of meticillin-resistant Staphylococcus aureus (MRSA). Int J Antimicrob Agents 37: 110-117, 2011.

20. Harris AD, Furuno JP, Roghmann MC, Johnson JK, Conway LJ, Venezia RA, Standiford HC, Schweizer ML, Hebden JN, Moore AC and Perencevich EN: Targeted surveillance of methicillin-resistant Staphylococcus aureus and its potential use to guide empiric antibiotic therapy. Antimicrob Agents Chemother 54: 3143-3148, 2010.

21. Vos MC, Behrendt MD, Melles DC, Mollema FP, de Groot W, Parlevliet G, Ott A, Horst-Kreft D, van Belkum A and Verbrugh HA: 5 years of experience implementing a methicillin-resistant Staphylococcus aureus search and destroy policy at the largest university medical center in the Netherlands. Infect Control Hosp Epidemiol 30: 977-984, 2009. 